\title{
'Through others we become ourselves': The dialectics of predictive coding and active inference
}

\author{
Dimitris Bolis ${ }^{1,2,3}$ \& Leonhard Schilbach ${ }^{1,2,4}$
}

1 Independent Max Planck Research Group for Social Neuroscience, Max Planck Institute of Psychiatry, Kraepelinstrasse 2-10, 80804 Munich-Schwabing, Germany

2 International Max Planck Research School for Translational Psychiatry (IMPRS-TP), Munich, Germany

3 Munich Medical Research School (MMRS), Dekanat der Medizinischen Fakultät, Ludwig-Maximilians-Universität München, Bavariaring 19, 80336 Munich, Germany

$4 \quad$ LVR Klinikum Düsseldorf / Kliniken der Heinrich-Heine-Universität Düsseldorf, Bergische Landstr. 2, 40629 Düsseldorf, Germany

dimitris_bolis@psych.mpg.de

leonhard_schilbach@psych.mpg.de

\begin{abstract}
Thinking Through Other Minds (TTOM) creatively situates the free energy principle within real-life cultural processes, thereby enriching both sociocultural theories and Bayesian accounts of cognition. Here, shifting the attention from thinking to becoming, we suggest complementing such an account by focusing on the empirical, computational and conceptual investigation of the multiscale dynamics of social interaction.
\end{abstract}

We applaud Veissière and colleagues for pursuing the ambitious goal of situating the free energy principle within the context of sociocultural processes (cf. TTOM; 2019). This is, indeed, a much needed undertaking, which has only recently started developing, holding promise for advancing not only relevant sociocultural research fields, but also computational psychiatry (cf. Friston and Frith, 2015; Bolis and Schilbach, 2017; 2018b; Gallagher and Allen, 2018; Constant et al., 2019). In fact, human cognition and culture have often been studied in isolation. For instance, the field of computational psychiatry has been developing rigorous experimental protocols and mathematical toolboxes to mechanistically explain human cognition and action. Yet, until recently a rather individualistic perspective has been adopted, which neglects levels of description beyond the individual (cf. De Jaegher and Di Paolo, 2007; Schilbach et al., 2013; Kirmayer and Crafa, 2014; Bolis et al., 2017). On the other hand, sociocultural fields, such as cultural anthropology, have rightfully adopted a more holistic perspective to complex 
phenomena of life, yet frequently lacking formal descriptions of cognitive and biological mechanisms (cf. Seligman and Brown, 2009).

An artificial dichotomy between the individual and the collective has inevitably led to a 'chicken-egg' paradox (cf. Dumas et al., 2014). However, such causality dilemmas dissolve once one considers the dialectical nature of human-becoming, which is multiscale, reciprocal, dynamic, cumulative and inherently contradictory (cf. Vygotsky 1930-1935/1978; Dumas et al., 2014; Bolis and Schilbach, 2018b; Di Paolo et al., 2018). Processes from evolution and culture to individual development, learning and sensorimotor activity, can all be viewed as mutually interacting adjustments between the species and the environment. Here, reciprocity is deep, as "it is not only humans who change the environment, but the environment in turn changes them in face of their impact on it" (Levins and Lewontin, 1985; Bolis and Schilbach, 2018b). TTOM, therefore, constitutes an important development because it addresses how human agents learn shared expectations and how they construct their own social niches in complex interaction between the individual and the environment.

We concretely appreciate the consideration of predictive coding and active inference within a framework of circular causality. Indeed, an organism can be viewed as embedded within the dialectic between the two above-mentioned processes, which in order to survive obeys a simple, but fundamental rule: "adjust yourself to reality or change the reality itself" (Friston, 2010; Bolis and Schilbach, 2018b). When it comes to TTOM, it is not only the agent which learns environmental regularities and adjusts accordingly, but the environment in turn 'learns' the agents' 'beliefs' through repeated and culturally regulated actions. TTOM resonates well with the dialectical attunement hypothesis (Bolis and Schilbach, 2018b), which views human-becoming as the interplay between internalization and externalization primarily within and due to culturally mediated social interaction, internalization being the "co-construction of bodily hierarchical models of the (social) world and the organism" [cf. predictive coding], while externalization the "collective transformation of the world" [cf. active inference]. In a nutshell, "interpersonal statistical regularities shape multiscale hierarchical models on an individual level and vice versa".

To offer a formal description of how environment 'learns', the authors interestingly suggest twisting the modeling equations by inverting relevant quantities across actions and sensations. This offers various potential modeling scenarios about the degree of interactivity within the system of brain-bodyenvironment-body-brain (cf. Froese et al., 2013). Here, a multiscale meta-Bayesian scheme might nicely 
lend itself for modeling not only individual processes, but also collective and environmental interactions (Bolis and Schilbach, 2017; Brandi et al., 2019; Ramstead et al., 2019).

Not only are we in line with the authors on conceptual and computational grounds, but also concerning the need for empirical studies. To make this more concrete, we describe certain experimental directions: Systematically varying social structure, cultural and socioeconomic background, affective bonds and interpersonal similarity across interacting individuals will enable the mechanistic study of interpersonal attunement. With regards to psychiatric disorders, construed as disorders of social interaction (Schilbach, 2016), two-person (or indeed collective) psychophysiology allows to move beyond the individual (cf. Bolis and Schilbach, 2018a). Taking autism as a paradigm example, the dialectical misattunement hypothesis has put forward a research line, which, moving away from an exclusive study of individual differences, considers types of interacting groups: i.e. autistic, neurotypical and mixed groups, expecting smoother interactions within the more homogenous groups or dyads (Bolis et al., 2017). Taken together, such experiments will not only inform TTOM within the 'neurotypical social world', but also open up avenues for evaluating and updating the ontological status of conditions, such as autism, as relational and interactional (cf. double empathy problem; Milton, 2012).

Apart from praising TTOM, we would also like to point out a fundamental aspect which, in our opinion, would benefit from further elaboration. We feel that the potentially constitutive role of real-time social interaction in sense-making and human-becoming was not sufficiently taken into account within the model (cf. De Jaegher and Di Paolo, 2007; Vygotsky 1930-1935/1978). It has been suggested that thinking about and with others might be fundamentally different in real-time interactive scenarios, as compared to passive observational situations (cf. second-person perspective; Schilbach et al., 2013; Redcay and Schilbach, 2019). Crucially, such interactive interpersonal processes have been thought of as dialectically preceding the individual both in evolutionary and developmental regards (cf. Bolis and Schilbach, 2018b; Tomasello, 2019). As Vygotsky proclaimed almost a century ago, "through others we become ourselves" (1931/1987). Yet, to do justice to the authors, the field today has not yet reached a conclusive consensus. For instance, while Di Paolo and colleagues (2018) suggest that "interactive situations present a richer, more complex set of possibilities" and "the key to our sociality is not in our heads or in our genes", Schönherr and Westra (2017) claim to have (conceptually) shown that "ersatz interactivity works just as well as the real thing", by 'real thing' denoting genuine, real-time social interaction. We, therefore, conclude our commentary with a question still desperately begging for a definite empirical answer. Does (real-time) social interaction matter... or is it all in our heads? 


\section{References}

Bolis, D., Balsters, J., Wenderoth, N., Becchio, C., \& Schilbach, L. (2017). Beyond autism: introducing the dialectical misattunement hypothesis and a bayesian account of intersubjectivity. Psychopathology, 50(6), 355-372.

Bolis, D., \& Schilbach, L. (2017). Beyond one Bayesian brain: Modeling intra-and inter-personal processes during social interaction: Commentary on "Mentalizing homeostasis: The social origins of interoceptive inference" by Fotopoulou \& Tsakiris. Neuropsychoanalysis, 19(1), 35-38.

Bolis, D., \& Schilbach, L. (2018a). Observing and participating in social interactions: action perception and action control across the autistic spectrum. Developmental cognitive neuroscience, 29, 168175.

Bolis, D., \& Schilbach, L. (2018b). 'I interact therefore I am’: The self as a historical product of dialectical attunement. Topoi, 1-14.

Brandi, M. L., Kaifel, D., Bolis, D., \& Schilbach, L. (2019). The Interactive Self-A Review on Simulating Social Interactions to Understand the Mechanisms of Social Agency. i-com, 18(1), 17-31.

Constant, A., Ramstead, M. J., Veissière, S. P., \& Friston, K. (2019). Regimes of Expectations: An Active Inference Model of Social Conformity and Decision Making. Frontiers in psychology, 10, 679.

De Jaegher, H., \& Di Paolo, E. (2007). Participatory sense-making. Phenomenology and the cognitive sciences, 6(4), 485-507.

Di Paolo, E. A., Cuffari, E. C., \& De Jaegher, H. (2018). Linguistic bodies: The continuity between life and language. MIT Press.

Dumas, G., Kelso, J. A., \& Nadel, J. (2014). Tackling the social cognition paradox through multi-scale approaches. Frontiers in psychology, 5, 882.

Friston, K. (2010). The free-energy principle: a unified brain theory?. Nature reviews neuroscience, 11(2), 127.

Friston, K., \& Frith, C. D. (2015). Active inference, communication and hermeneutics. Cortex, 68, 129143.

Froese, T., lizuka, H., \& Ikegami, T. (2013). From synthetic modeling of social interaction to dynamic theories of brain-body-environment-body-brain systems. Behav. Brain Sci, 36(4), 420-421.

Gallagher, S., \& Allen, M. (2018). Active inference, enactivism and the hermeneutics of social cognition. Synthese, 195(6), 2627-2648. 
Kirmayer, L. J., \& Crafa, D. (2014). What kind of science for psychiatry?. Frontiers in Human Neuroscience, 8, 435.

Levins, R., Lewontin, R. C. (1985). The dialectical biologist. Harvard University Press, Cambridge

Milton, D. E. (2012). On the ontological status of autism: the 'double empathy problem'. Disability \& Society, 27(6), 883-887.

Ramstead, M. J. D., Badcock, P. B., \& Friston, K. J. (2018). Answering Schrödinger's question: A freeenergy formulation. Physics of life reviews, 24, 1-16.

Redcay, E., \& Schilbach, L. (2019). Using second-person neuroscience to elucidate the mechanisms of social interaction. Nature Reviews Neuroscience, 1.

Schilbach, L. (2016). Towards a second-person neuropsychiatry. Philosophical Transactions of the Royal Society B: Biological Sciences, 371(1686), 20150081.

Schilbach, L., Timmermans, B., Reddy, V., Costall, A., Bente, G., Schlicht, T., \& Vogeley, K. (2013). Toward a second-person neuroscience 1. Behavioral and brain sciences, 36(4), 393-414.

Schönherr, J., \& Westra, E. (2017). Beyond 'Interaction': How to Understand Social Effects on Social Cognition. The British Journal for the Philosophy of Science, 70(1), 27-52.

Seligman, R., \& Brown, R. A. (2009). Theory and method at the intersection of anthropology and cultural neuroscience. Social cognitive and affective neuroscience, 5(2-3), 130-137.

Tomasello, M. (2019). Becoming human: A theory of ontogeny. Belknap Press.

Veissière, S. P., Constant, A., Ramstead, M. J., Friston, K. J., \& Kirmayer, L. J. (2019). Thinking Through Other Minds: A Variational Approach to Cognition and Culture. Behavioral and Brain Sciences, 197.

Vygotsky, L. S. (1987). The genesis of higher mental functions. In R. Reiber (Ed.), The history of the development of higher mental functions (Vol. 4, pp. 97-120). New York: Plennum. Original work 1931.

Vygotsky, L. S. (1978). Mind in society: the development of higher psychological processes. Harvard University Press. Original work 1930-1935. Translated in Greek, (1997) by A. Bibou and S Vosniadou, Gutenberg. 